\title{
A survey of the Siberio-Nearctic genus Masikia Millidge, 1984 (Aranei: Linyphiidae: Erigoninae)
}

\section{Обзор сибирско-неарктического рода Masikia Millidge, 1984 (Aranei: Linyphiidae: Erigoninae)}

\author{
Anna A. Nekhaeva ${ }^{1}$, Yuri M. Marusik ${ }^{2,3}$, Don Buckle ${ }^{4}$ \\ А.А. Нехаева ${ }^{1}$, Ю.М. Марусик ${ }^{2,3}$, A. БакА ${ }^{4}$
}

\footnotetext{
A.N. Severtsov Institute of Ecology and Evolution, Russian Academy of Sciences, Leninsky prospekt 33, Moscow 119071, Russia. E-mail: adrealinea@gmail.com

${ }^{2}$ Institute for Biological Problems of the North RAS, Portovaya Str. 18, Magadan 685000, Russia. E-mail: yurmar@mail.ru

${ }^{3}$ Department of Zoology \& Entomology, University of the Free State, Bloemfontein 9300, South Africa.

${ }^{4}$ 16-3415 Calder Crescent, Saskatoon, SK, S7J 5A3 Canada.

' Институт проблем экологии и эволюции им. А.Н. Северцова РАН, Ленинский проспект 33, Москва 119071, Россия.

${ }^{2}$ Институт биологических проблем Севера, ДВО РАН, Портовая 18, Магадан 685000, Россия.
}

KEY WORDS: Araneae, Erigone-group, new species, Arctic, tundra zone, Russia, Alaska, Canada, sea marshes.

КЛЮЧЕВЫЕ СЛОВА: Araneae, группа Erigone, новый вид, Арктика, тундровая зона, Россия, Аляска, Канада, приморские марши.

ABSTRACT: Masikia Millidge, 1984 was known to encompass two species prior to this study. The present review reveals three distinct morphospecies all represented by both sexes: $M$. indistincta (Kulczyński, 1908), M. caliginosa Millidge, 1884 and M. bizini sp.n. Generic alignment of $M$. relicta (Chamberlin, 1949) known from female holotype from Vermont is doubtful. Masikia caliginosa Millidge, 1884 stat.rev. is removed from synonymy with $M$. indistincta. Its male is described for the first time. All three species are illustrated in detail and their distribution is mapped. In several localities $M$. caliginosa and $M$. indistincta were found to be sympatric.

How to cite this article: Nekhaeva A.A., Marusik Yu.M., Buckle D. 2019. A survey of the Siberio-Nearctic genus Masikia Millidge, 1984 (Aranei: Linyphiidae: Erigoninae) // Arthropoda Selecta. Vol.28. No.1. P.157-168. doi: 10.15298/arthsel. 28.1.15

РЕЗЮМЕ: Род Masikia Millidge, 1984 до настоящего исследования включал всего два вида: $M$. indistincta (Kulczyński, 1908) (север Голарктики, О70) и M. relicta (Chamberlin, 1949) (Вермонт, США, ,). Ревизия доступных коллекционных материалов позволила выявить ещё два вида: M. caliginosa Millidge, 1884 (север Голарктики, +) и M. bizini sp.n. (северное Охотоморье, $\bigcirc^{\top}$ ). Masikia caliginosa Millidge, 1884 stat. rev. исключён из синонимии с M. indistincta, а его самцы описаны впервые. Все три вида, встречающиеся в Палеарктике, проиллюстрированы, приведены данные об их распространении. В некоторых локалитетах M. caliginosa и $M$. indistincta обитают симпатрично. Родовая принадлежность M. relicta (Chamberlin, 1949), известного по самке, сомнительна.

\section{Introduction}

Masikia Millidge, 1984 is a small genus of Erigoninae spiders. It was described for two species, $M$. atra Millidge, 1984 and M. calignosa Millidge, 1984 known exclusively from females and collected from Arctic Canada and Alaska. In the first review of the genus, Eskov \& Marusik [1994] synonymized both species with Masikia indistincta (Kulczyński, 1908), a species originally described in Macrargus from northern Yakutia, and described the previously unknown male. In the same work, Montilaira reclicta Chamberlin, 1949 from Vermont was transferred to Masikia. The discovery of the male of $M$. indistincta allowed the recognition of relationships of the genus and it was assigned by Eskov \& Marusik [1994] to the Erigone-group of genera sensu Millidge [1977]. Currently Masikia includes two valid species [WSC, 2019]: M. indistincta and M. relic$t a$. While identifying material collected in the Arctic for several ecological projects, we found males of two morphotypes, sometimes these males were taken from the same sample. The males differed in the number and thickness of cephalic setae and also in the shape of their tibial apophyses and embolic division. This led us to the necessity of providing a revision of all available material of this species. Analysis of this material (over 600 specimens) revealed two distinct morphospecies occurring both in the Nearctic and Palaearctic, and 
often sympatrically. We also found a third morphospecies, collected recently on sea marshes in northern Cisokhotia. The solution of some nomenclatorial problems and clarification of the systematic relationships of the genus, as well as the morphological description of the three species and mapping their distributions, is the goal of the paper.

\section{Material and methods}

Specimens were photographed at the Zoological Museum (University of Turku, Finland) with a Canon EOS 7D camera attached to an Olympus SZX16 stereomicroscope and a SEM JEOL JSM-5200 scanning microscope. Digital images were montaged using CombineZP and Helicon focus 3.10 image stacking software. Epigynes were cleared in a $\mathrm{KOH} /$ water solution until soft tissues were dissolved. Photographs were taken in dishes with paraffin on the bottom holding the specimens in place. All measurements are given in millimeters. Lengths of carapace and leg segments were measured on the dorsal side.

Abbreviations of the leg segments: $\mathrm{Fe}$ - femur, $\mathrm{Me}-$ metatarsus, $\mathrm{Pa}$ - patella, Ta - tarsus, Ti - tibia.

Abbreviation of the museums: CBGG - Centre for Biodiversity Genomics, University of Guelph, Canada; DJB Donald J. Buckle, personal collection, Saskatoon, Canada; LEM - Lyman Entomological Museum, McGill University, Montreal, Canada; MMUM - the Manchester Museum, the University of Manchester, UK; ZMMU — Zoological Museum of the Moscow State University, Russia; ZMUT Zoological Museum of the University of Turku, Finland.

\section{Taxonomic survey}

\section{Masikia Millige, 1984}

Masikia Millidge, 1984: 152.

Masikia: Eskov, Marusik, 1994: 47.

TYPE SPECIES. Masikia atra Millidge, 1984 (=M. indistincta (Kulczyński, 1908)).

COMMENTS. The genus was considered and discussed only in the two papers listed above. The original genus description is lacking proper diagnosis and comments about relationships of the genus. Eskov \& Marusik [1994] indicated that genus belongs to the Erigone-group of genera sensu Millidge [1977] and seems related to Collinsia O. PickardCambridge, 1913.

DIAGNOSIS. Masikia differs from the other genera of the Erigone-group by having a modified cephalic part bearing a set of strong setae directed anteriorly (character unknown in the other genera), rounded and posteriorly directed tip of embolus and triangle shaped epigyne with copulatory opening located posteriorly and hidden by the epigynal plate.

DESCRIPTION. Medium-sized (body length 1.85-2.33, carapace $0.68-1.1$ long and $0.63-0.83$ width in $\sigma^{7} \sigma^{7}$, and $1.75-2.53,0.75-1.13$ and $0.60-0.8$ in +9 respectively), dark colored erigonines, carapace without postocular pits, moderately elevated behind the eye area, elevation provided with a few (2-6) robust setae arranged in a line or compact bundle. The ocular area with arch-shaped row of 6 to 10 setae around PME. Chelicera unmodified with ca. 40-50 stridulatory ridges. Tibial spines 2222 or 2221, Tm I 0.47-
0.62, Tm IV absent. Abdomen unmodified, concolorous. Palpal tibia with two trichobothria.

Copulatory organs. Male palp: palpal tibia moderately elongated, more or less vertical, wider than long, with a small subtriangular or rounded retrolateral apophysis and a broad dorsal apophysis subdivided into 2 branches; retrolateral margin with row of 4-6 strong setae. Paracymbium medium-sized, T-shaped, tip C-shaped. Tegulum wide, vertical. Suprategulum with a long, pointed, membranous apophysis. Embolic division complex, with terminal apophysis ( $T a$ ) partly hidden by embolic outgrowth $(E p)$ and clawshaped embolus bearing short stylus in 2 species (lacking in $M$. bizini sp.n.) and a flattened tooth $(T t)$ of the tailpiece (Tp). Embolic outgrowth with 2 sharply pointed processes. Embolus bent prolaterally with rounded base imbedded into a notch of radix $(R n)$ in $M$. caliginosa and $M$ bisizni sp.n.

Epigyne slightly protruding, triangular, with a posteriorly situated orthogonal median plate $(M p)$. Sides of epigyne with long setae, as long as protruding part of the epigynal plate. Median plate subdivided into 2 parts by transverse furrow $(T f)$. Vulva with medium-sized oviform receptacles and moderately long, slightly curved copulatory ducts.

COMMENT. Two widespread species, $M$. indistincta and $M$. caliginosa are highly variable in size of carapace and overlapping in carapace length/width ratio (Fig. 9). However, $M$. indistincta in general is smaller and has a thinner carapace. Males of $M$ bisizni sp.n. are larger than the two other species (Fig. 9B).

DISTRIBUTION. The genus has an almost circum-Holarctic distribution but is absent in Europe, except the most northwesternmost part. The two species $M$. indistincta and M. caliginosa have almost the same range (Map 1). Masikia caliginosa is known in the Nearctic from the extreme western arctic only while $M$. indistincta has been found across arctic Canada. Both species are restricted to the tundra zone. The other two species, $M$. bizini sp.n. and $M$. relicta, are known only from their type localities. The former was found in seashore marshes in the boreal zone, the latter from alpine tundra on an isolated mountain top in the northeastern USA.

Masikia indistincta (Kulczyński, 1908)

Figs 1E, 2A-E, 3A-C, 4H-J, 5A-D, 6G-H, 7A-C; 8A-F, 9, Map 1. (†).

Macrargus indistinctus Kulczyński, 1908: 34, Tab. 1, fig. 27

Masikia atra Millidge, 1984: 154, Figs 125, 127, 129 (†).

Masikia indistincta: Eskov, Marusik, 1994: 47, figs 27-33 ( $\sigma^{7}$, transferred to Masikia, synonymized with M. atra and $M$. caliginosa, first description of the $\sigma^{7}$ ); Marusik et al., 2016: 1718, figs. $11-17\left(\mathrm{O}^{\top}+\right)$.

MATERIAL EXAMINED. RUSSIA: Arkhangelsk Area: $3+0$ (ZMMU), Novaya Zemlya Archipelago, Malye Karmakuly, env. of Polar Station, 21.07-1.08.2015 (V.M. Spitsyn). Nenets Autonomous Okrug: 4우 (ZMMU), Pakhancheskaya Guba, Matyui-Salya Cape, $68^{\circ} 31^{\prime} \mathrm{N} 57^{\circ} 19.5^{\prime} \mathrm{E}, 25.07-5.08 .2015$ (O.L. Makarova, M.S. Bizin); $50^{\top} \sigma^{\top} 10$ + $\bigcirc$ (ZMMU), Yugorskii Peninsula, env. of Amderma, 69.76 N, 61.67 E, sea marshes, 4-8.07.2018 (M.S. Bizin, B.D. Efeikin); $3800^{\top} \sigma^{\top}$ to (ZMMU, MMUM), Barents Sea, Dolgiy Island, $69^{\circ} 12^{\prime} \mathrm{N} 59^{\circ} 13^{\prime} \mathrm{E}$, Nenets Reserve, 6-28.07.2004 (O.L. Makarova); $10^{7}$ (ZMMU), Vaigach Island, env. of Varnak Vil., 69. $69233^{\circ} \mathrm{N} 060.16689^{\circ} \mathrm{E}$, seashore, algae debris and under logs, 8.07.2015 (A.B. Babenko); $10^{7} 1$ 1 (ZMMU), Yugorskii Peninsula, env. of Polar Station Yu-Shar, 2-6.07.1983, (V.I. Bulavintzev). Yamalo-Nenets Autonomous Okrug: $4 \sigma^{7} \sigma^{\top} 10$ 10 (ZMMU), Yamal Peninsula, env. of Sabetta Vil., Sabetta R., $71^{\circ} 14^{\prime} 36.9^{\prime \prime} \mathrm{N}$ 

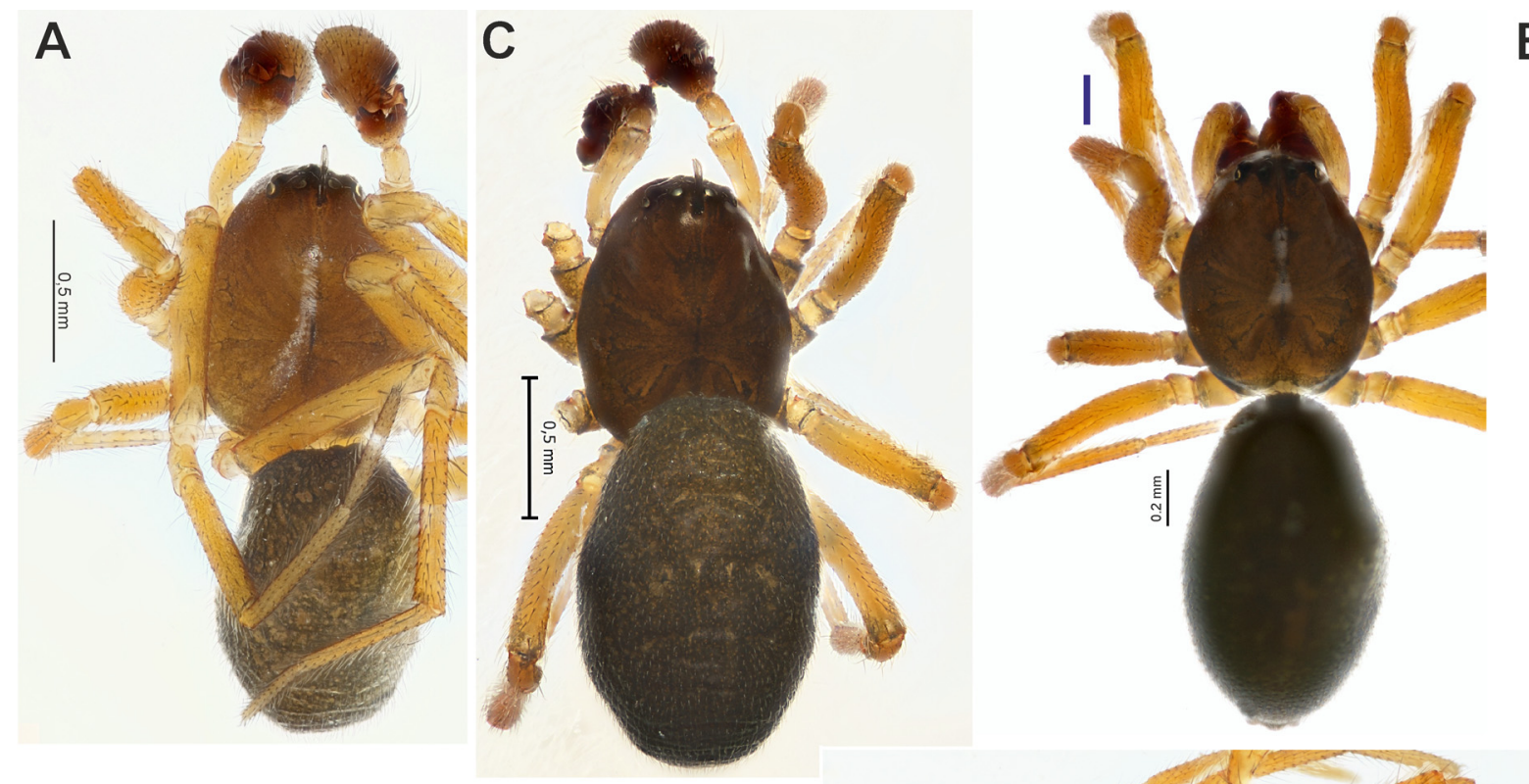

E
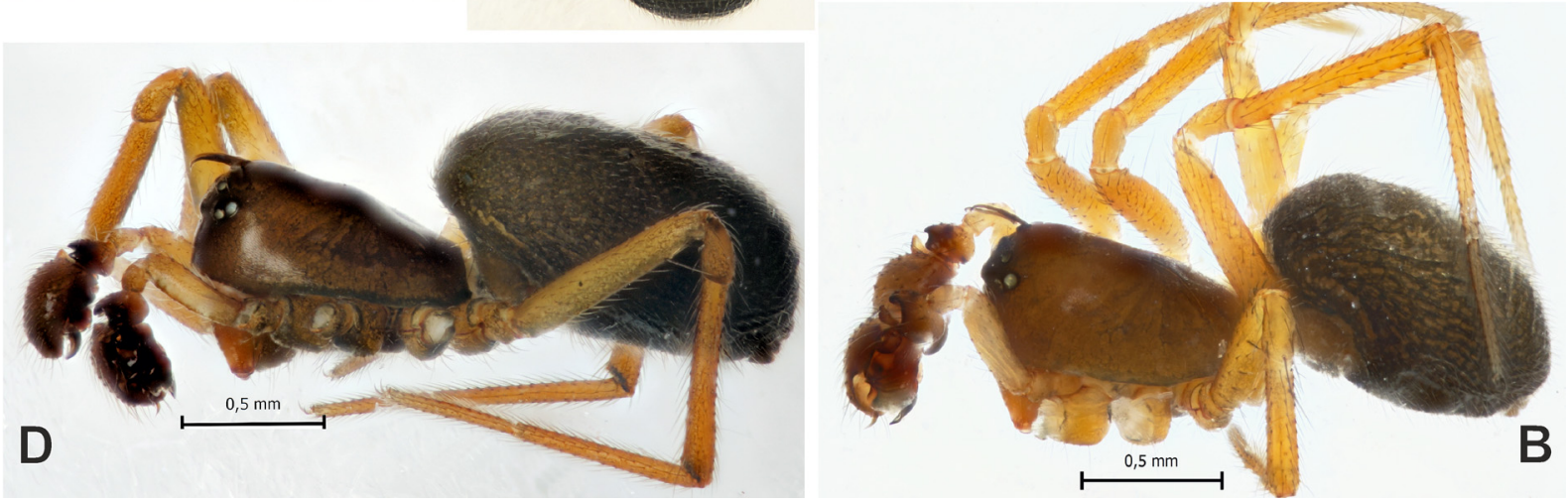

Fig. 1. Habitus of male of Masikia bizini sp.n. (A, B), M. caliginosa (C, D) and M. indistincta (E). A, C, E - dorsal; B, D - lateral. Рис. 1. Внешний вид самца Masikia bizini sp.n. (А, В), M. caliginosa (С, D) и M. indistincta (Е). А, С, Е — сверху; В, D — сбоку.

$71^{\circ} 41^{\prime} 47^{\prime \prime} \mathrm{E}, 15.08 .2014$ (L.B. Rybalov); $350^{7} 0^{7} 48$ 우 (ZMMU), same locality, July 2017 (L.B. Rybalov, A.I. Bastrakov); 8우 (ZMMU), S Yamal Peninsula, env. of Shchuchye Vil., edge of oligotrophic bog, Polytrichum, 24.08 .1980 (A.L. Tikhomirova, E.M. Veselova); $10^{7} 3$ 우 (ZMMU), Gydan Peninsula, Tazovskii District, $72.19228^{\circ} \mathrm{N} 77.57839^{\circ} \mathrm{E}$, sea marshes, $13-18.07 .2016$ (S.B Rozenfeld); $20^{7} \sigma^{7}$ (ZMMU), same district, Shokalsky Isl., $72^{\circ} 58^{\prime} \mathrm{N}$ $74^{\circ} 27^{\prime} \mathrm{E}$, sea marshes, sifting, 8.08.2016 (A.A. Nekhaeva), Krasnoyarsk Province, $30^{7} 0^{\top} 2+\bigcirc$ (ZMMU), NW Taimyr Peninsula, 40 $\mathrm{km} \mathrm{S}$ from Dikson Vil., Efromova R., $73.2006^{\circ} \mathrm{N} 80.8198^{\circ} \mathrm{E}$, moss, July 2003 (D.V. Osipov); 4우 (ZMMU), N coast of Taimyr Lake, Ozhidaniya Bay, $74^{\circ} 36^{\prime} \mathrm{N} 101^{\circ} 44^{\prime} \mathrm{E}$, July 1993 (A.B. Babenko); $10^{7}$ (ZMMU), NW part of Taimyr Peninsula, Ragozinka River [72 $\left.{ }^{\circ} 57^{\prime} \mathrm{N} \mathrm{80} 56^{\prime} \mathrm{E}\right]$, August 1982 (Yu.I. Chernov); 1웅, (ZMMU), same region, Syradasai River, August 1982 (Yu.I. Chernov); $30^{7} \mathrm{O}^{7}$ 4오 (ZMMU), Taimyr Nature Reserve, Byrranga Mts., 27.07.1992 (A.B. Ryvkin). Yakutia: $4 \bigcirc^{7} 0^{7} 4$ 웅 (ZMMU), Ust-Lenskiy Reserve, env. of Tyylaakh, $72.194709^{\circ} \mathrm{N} 128.077528^{\circ} \mathrm{E}, 5-16.07 .2015$ (A.V. Tanasevitch, A.A. Nekhaeva). Chukotka: $10^{7} 5$ 우 (ZMMU), NW part, Apapelgino, $69^{\circ} 48^{\prime} 44^{\prime \prime} \mathrm{N} 170^{\circ} 36^{\prime} 23^{\prime \prime} \mathrm{E}, 13-24.07 .2018$ (K.V. Makarov, O.L. Makarova); $170^{7} 0^{\top} 11$ 웅 (ZMMU), Wrangel Isl., Gusinaya R. down flow, 1984 (O.A. Khrulyova); $10^{7} 1$ \% (ZMMU), same island, Neizvestnaya R. middle flow, mountain terrace, willow-sedge-moss tussocks, 23.08 .1983 (O.A. Khrulyova); 2 오 (ZMMU), same island, Western part, upper reaches of Neozhidannya R., $71.0602^{\circ} \mathrm{N}, 178.921^{\circ} \mathrm{E}$, wet river valley with sedge-moss vegetation, pitfall traps, 1.08.2016 (L.F. Volkova); $10^{7}$
1 ( Range, $71.0046^{\circ} \mathrm{N}, 179.518^{\circ} \mathrm{W}$, wet base of ridge with sedgemoss-sphagnum vegetation, 18.07.2015 (O.A. Khruleva); $70^{7} 0^{7}$ 3 우 (ZMMU), Chukotka Peninsula, N Koluchenskaya Guba, Belyaka Spit, $67^{\circ} 03^{\prime} \mathrm{N} 174^{\circ} 37^{\prime} \mathrm{W}$, bogged depression between with sedges, green mosses and sphagnum cover, 19-27.06.2015 (N.I. Vartanyn, A.G. Dondua); $30^{7} 0^{7} 5$ ㅇ (ZMMU), Lavrentiya Vil., $65^{\circ} 35^{\prime} \mathrm{N} 171^{\circ} 02^{\prime} 28^{\prime \prime} \mathrm{W}$, sedge-moss bog, 21.07 .2013 (A.V. Tanasevitch); $10^{7}$ (ZMMU), NE Siberia, Chukotka, Provideniya Bay, bog, 21.07.1972 (A.L. Tikhomirova, V.A. Turchaninova).

CANADA: Northwest Territories: $170^{7} 0^{7} 11$ 오 (DJB), Banks Island, $73.23^{\circ} \mathrm{N}, 119.55^{\circ} \mathrm{W}, 7-19.07 .2011$ (Northern Biodiversity Program). Nunavut: $10^{7}$ (LEM), Cambridge Bay, Victoria Island, $68.113^{\circ} \mathrm{N}, 105.422^{\circ} \mathrm{W}, 11-15$ June 2011 (Northern Biodiversity Program); 19 (CBGG), Eastbay, Southampton Island, $64^{\circ} \mathrm{N}, 82.5^{\circ} \mathrm{W}$, 22.06.2008 (E. Bolduc, M. Patenaude); $10^{7}$ (CBGG), 19 (LEM) Iqaluit, Baffin Island, $63.757^{\circ} \mathrm{N}, 68.568^{\circ} \mathrm{W}, 21-25$ July 2010 (Northern Biodiversity Program); Qikiqtaaluk, Western Bylot Island, Bylot Camp, $73.147^{\circ} \mathrm{N}, 79.981^{\circ} \mathrm{W}, 19.06 .2008$ (E. Bolduc, M. Patenaude). Yukon Territory: $20^{7} O^{7} 3$ 우 (CBGG), Hershel Island, $69.571^{\circ} \mathrm{N}, 138.902^{\circ} \mathrm{W}, 15.06 .2007$ (D. Reid, M. Leung, S. Gilbert, G.-O. Cimon).

NOTE. Two sibling species with very similar epigynes, $M$. indistincta and $M$. caliginosa co-occur in the type locality of $M$. indistincta. We recognized $M$. indistincta thanks to the outline of the epigyne given by Kulczyński [1908: fig. 27] which matches well with our specimens (cf. Fig. 7C). 

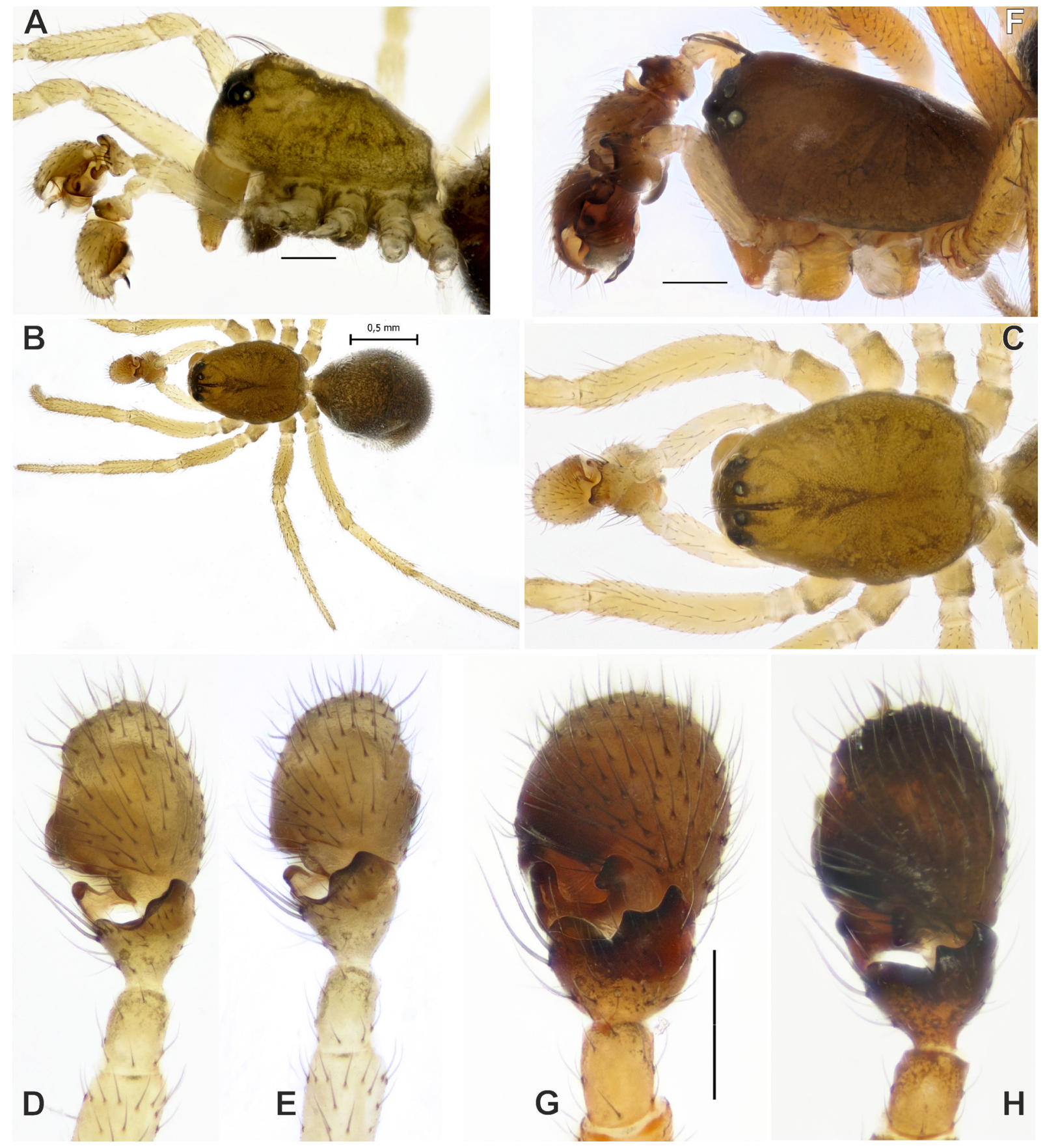

Fig. 2. Habitus and male palps of M. indistincta (A-E - Yamal Pnsl.), M. bizini sp.n. (F, G) and M. caliginosa (H - Shokalskogo Isl.). A, F - prosoma, lateral; B - body, dorsal; C - prosoma, dorsal; D, E, G, H - palp, dorsal. Scale $=0.2 \mathrm{~mm}$, if not otherwise indicated.

Рис. 2. Внешний вид и пальпы самцов M. indistincta (A-E - п-ов Ямал), M. bizini sp.n. (F, G) and M. caliginosa (H - o. Шокальского). A, F — головогрудь, сбоку; В — тело, сверху; C — головогрудь, сверху; D, E, G, H — пальпа, сверху. Масштаб 0,2 мм, если не обозначено иное.

DIAGNOSIS. Males of $M$. indistincta differ significantly from congeners by their weaker cephalic setae which are longer than the clypeus and arranged in a row. Males of $M$. indistincta have fewer stridulatory ridges than $M$. caliginosa (40 vs. 50). Palp differs from that of congeners by the non-rounded ventral margin of tibia ( $v s$. rounded), shal- low retrolateral apophysis and shallow notch of the dorsal apophysis ( $v s$. triangle shaped and deeper notch), relatively long stylus of embolus, and embolus not imbedded into notch of radix.

Females of $M$. indistincta are very similar to those of $M$. caliginosa and can be separated by their less protruding 

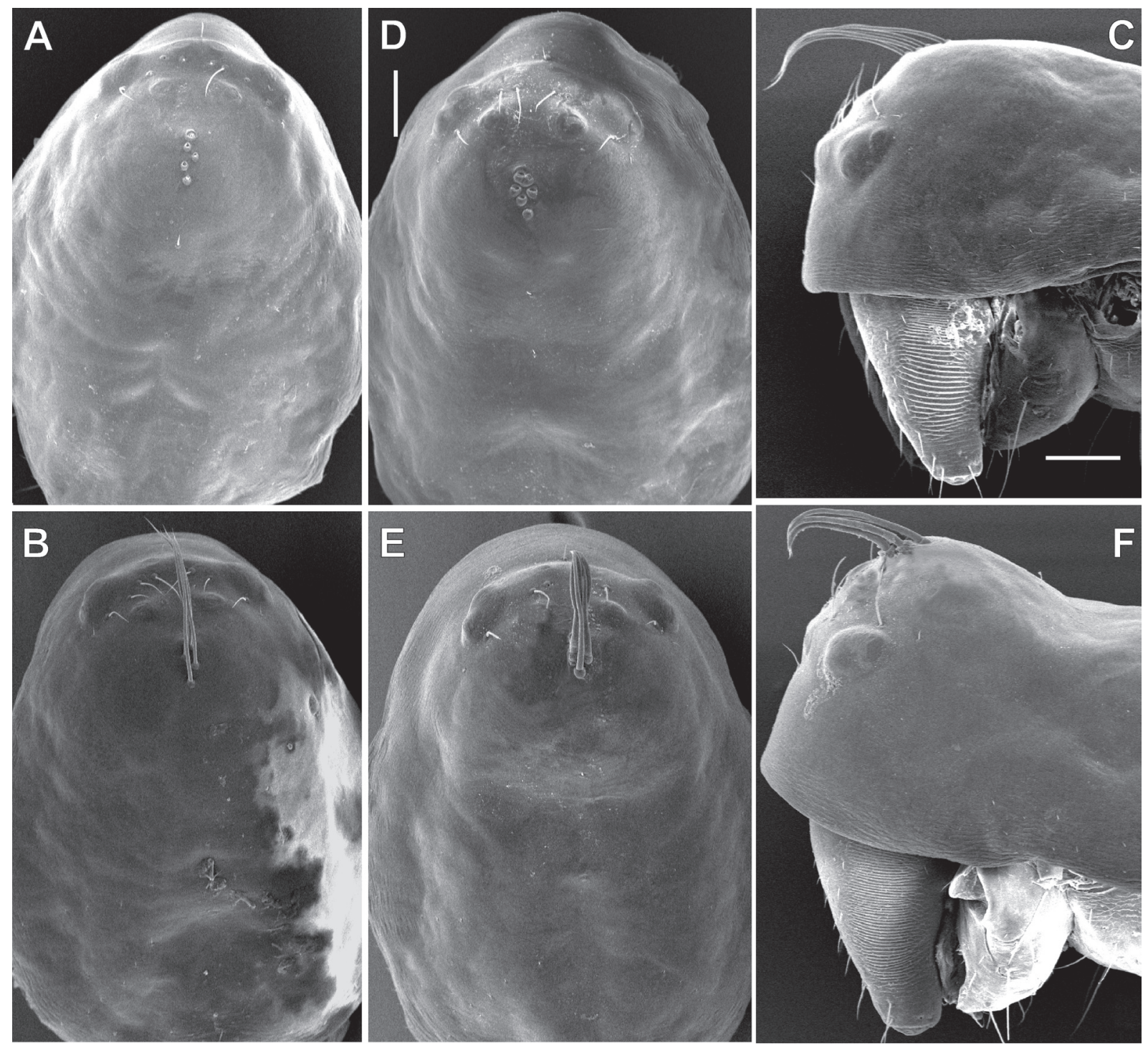

Fig. 3. Male prosoma of Masikia indistincta (A-C - from Dolgiy Isl.) and M. caliginosa (D-F - from Shokalsky Isl.). A, D carapace with removed cephalic macrosetae; B, E - same, intact; C, F - prosoma, lateral. Scale $=0.1 \mathrm{~mm}$.

Рис. 3. Головогрудь самца Masikia indistincta (A-C - o. Долгий) и M. caliginosa (D-F - о. Шокальского). A, D - карапакс, щетинки удалены; В, Е — то же, интактный экземпляр; C, F — головогрудь, сбоку. Масштаб 0,1 мм.

epigynal plate (ratio width/length 3 vs. 2) and broader median plate (as long as wide $v s$. longer than wide).

DESCRIPTION. Total $\sigma^{7} / 9$ length $1.85-1.93 / 1.75-$ 2.53. Carapace brownish, length/width $0.85-0.93 / 0.63$ 0.70 in $\sigma^{7}, 0.78-1.13 / 0.60-0.68$ in + . Male cephalic part with 5 strong macrosetae arranged in a row, ocular area with 9-10 small setae arranged in arch-shaped row (Fig. $3 \mathrm{~A}-\mathrm{C})$. Legs yellowish grey, length of joints as shown below; tibial spines 2222 or 2221 (distal spine of TiIV relatively short, in some specimens absent), TmI 0.47
0.58 . Abdomen grey. Male chelicera with about 40 stridulatory ridges (Fig. 3C).

Male palp as in Figs 2D-E, 4H-J, 5A-D, 6G-H. Tibia with almost straight ventral margin; retrolateral apophysis shallow; dorsal apophysis with shallow notch. Embolus not imbedded into notch of radix, stylus long (as wide as terminal part of embolus).

Epigyne as in Figs 7A-C, 8A-F. Plate with small protrusion (width/length ratio about $3 / 2$ ). Median plate variable in shape, as long as wide.

Masikia indistincta leg lengths.

\begin{tabular}{ccccccc}
\hline $\mathrm{O}^{7} / P$ & $\mathrm{Fe}$ & $\mathrm{Pa}$ & $\mathrm{Ti}$ & $\mathrm{Me}$ & $\mathrm{Ta}$ & Total \\
\hline I & $0.63 / 0.63$ & $0.20 / 0.23$ & $0.45 / 0.43$ & $0.48 / 0.43$ & $0.40 / 0.38$ & $2.16 / 2.10$ \\
II & $0.60 / 0.53$ & $0.20 / 0.20$ & $0.40 / 0.38$ & $0.45 / 0.40$ & $0.38 / 0.45$ & $2.03 / 1.96$ \\
III & $0.48 / 0.48$ & $0.15 / 0.20$ & $0.38 / 0.38$ & $0.40 / 0.40$ & $0.30 / 0.33$ & $1.71 / 1.79$ \\
IV & $0.73 / 0.68$ & $0.23 / 0.20$ & $0.68 / 0.63$ & $0.63 / 0.58$ & $0.45 / 0.38$ & $2.72 / 2.47$ \\
\hline
\end{tabular}



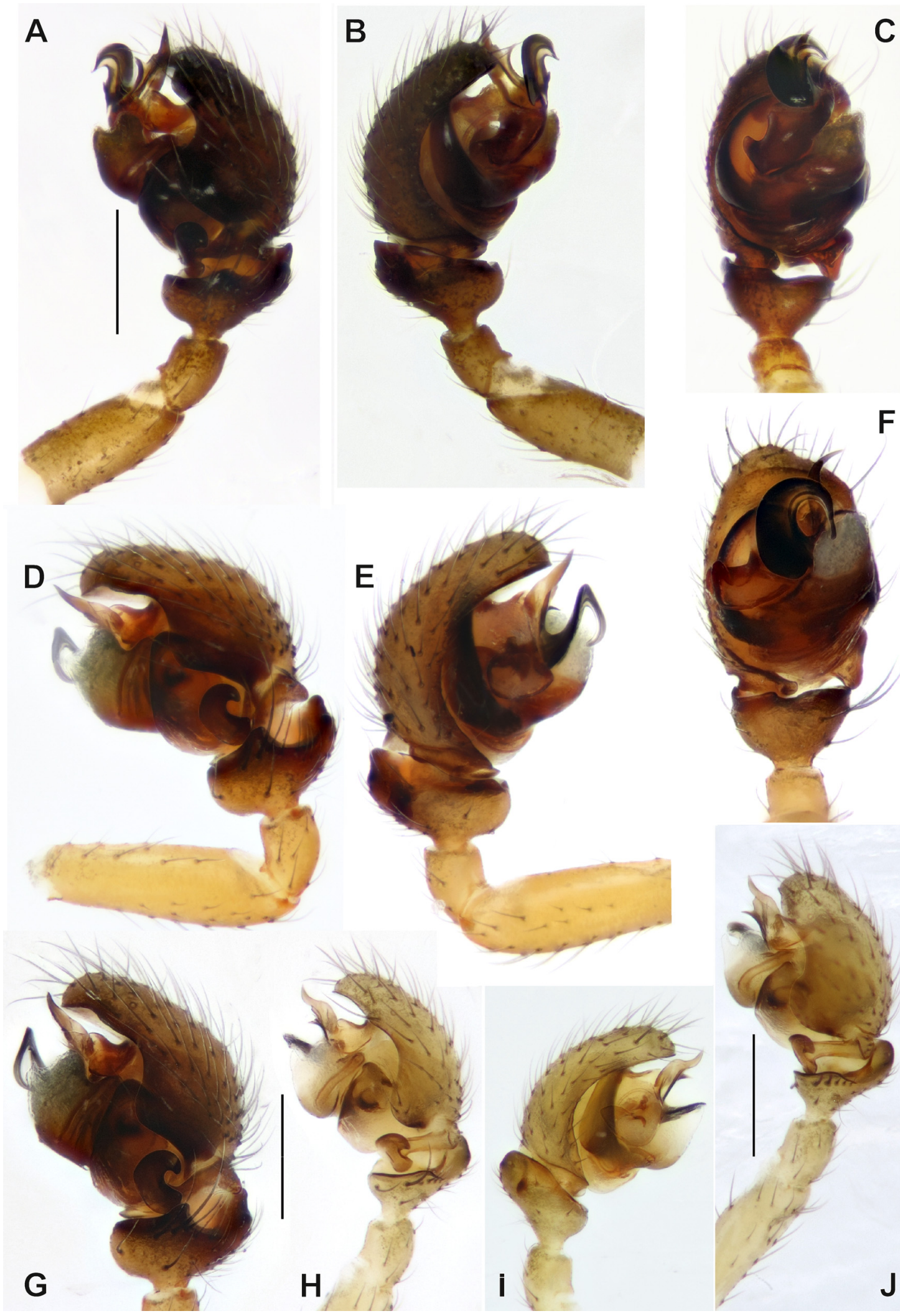

$\mathbf{F}$
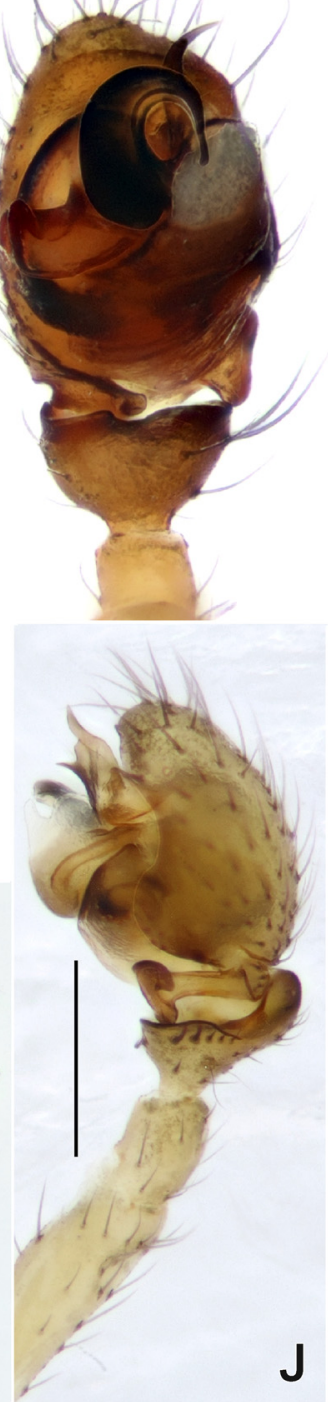

Fig. 4. Male palp of Masikia caliginosa (A-C - Shokalsky Isl.), M. bizini sp.n. (D-G) and M. indistincta (H-J - Yamal Pnsl.). A, D, $\mathrm{G}, \mathrm{H}, \mathrm{J}$ - retrolateral; B, E, I - prolateral; C, F - ventral. Scale $=0.2 \mathrm{~mm}$ (same for all figures).

Рис. 4. Пальпа самца Masikia caliginosa (А-C - o. Шокальского), M. bizini sp.n. (D-G) и M. indistincta (Н-J — п-ов Ямал). А, D, G, H, J - ретролатерально; В, E, I - пролатерально; C, F - снизу. Масштаб 0,2 мм (для всех рисунков). 

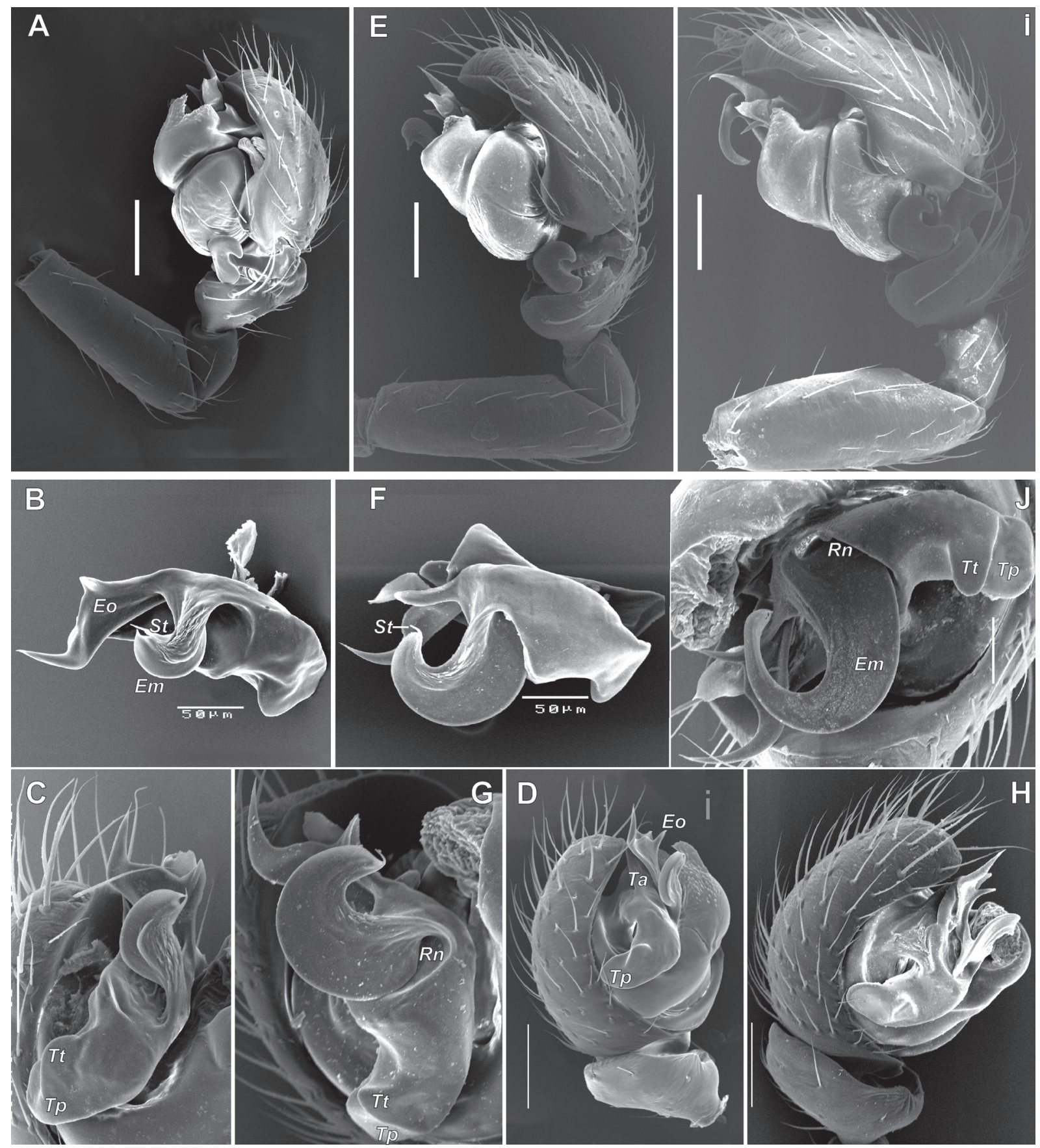

Fig. 5. Male palp of Masikia indistincta (A-D - from Dolgiy Isl.), M. caliginosa (E-H - from Shokalski Isl.) and M. bizini sp.n. (IJ). A, E, I - whole palp, retrolateral; B, F - dissected embolic division, ventral; C, G, J - palp, ventral, showing embolic division; D, H prolateral. Scale $=0.1 \mathrm{~mm}$, if not otherwise indicated.

Рис. 5. Пальпа самца Masikia indistincta (A-D - о. Долгий), M. caliginosa (E-H - о. Шокальского) и M. bizini sp.n. (I-J). А, Е, I - вся пальпа, ретролатерально; В, F - отделенный эмболюсный отдел, снизу; C, G, J — пальпа, снизу, показан эмболюсный отдел; D, Н - пролатерально. Масштаб 0,1 мм, если не обозначено иное.

HABITATS. This species is restricted to the tundra zone and is especially numerous on sea marshes and lake shores [Marusik et al., 2016].

DISTRIBUTION. The species is known from Pechora
Sea (ca. $57^{\circ} \mathrm{E}$ ) to Baffin Island in the Canadian Arctic Archipelago (ca. $80^{\circ} \mathrm{W}$ ), north to $74.6^{\circ} \mathrm{N}$ in Taimyr Peninsula and south to $64.5^{\circ}$ in Chukotka Peninsula and $64^{\circ} \mathrm{N}$ in Southampton Island (Map 1). 

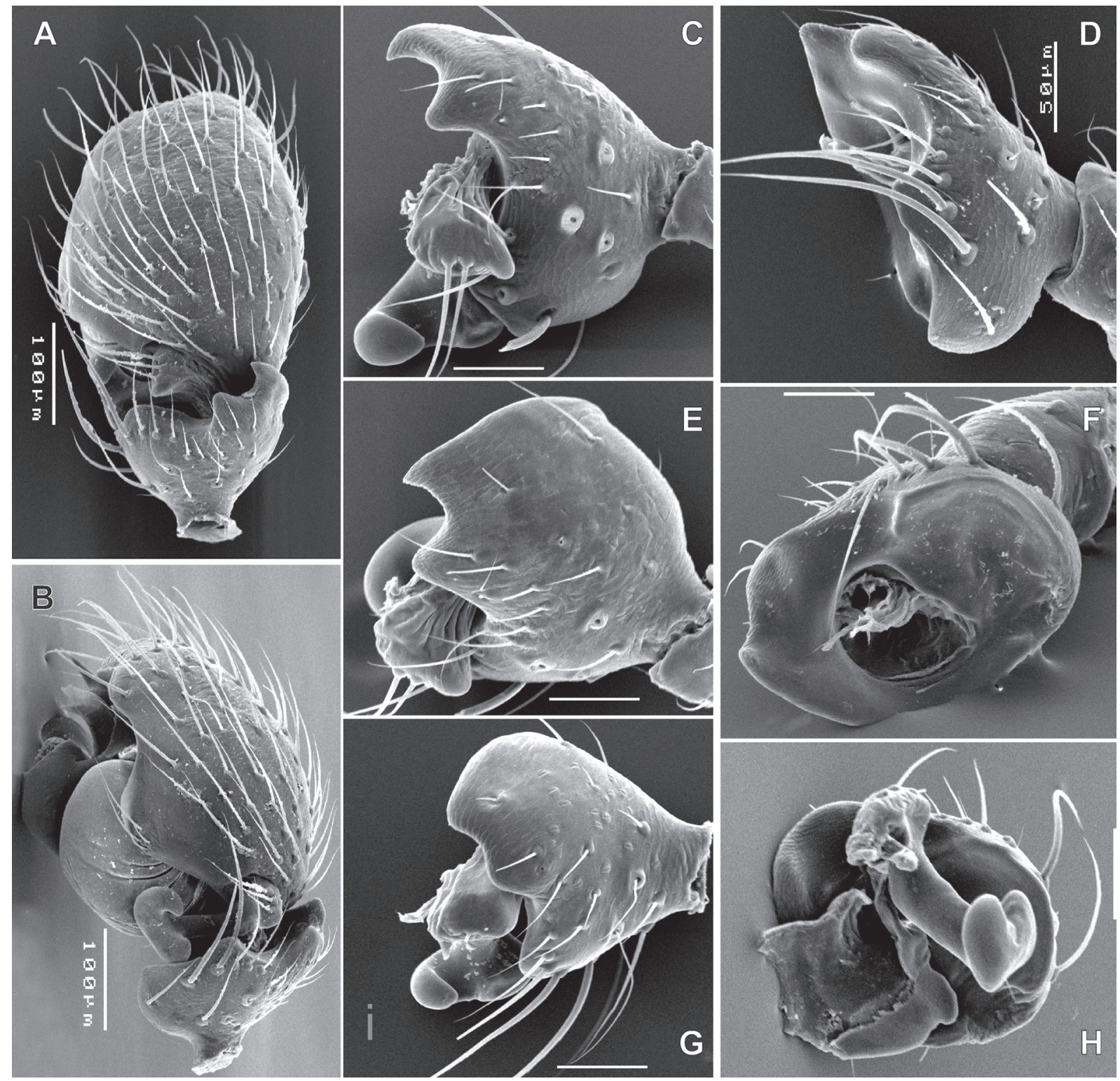

Fig. 6. Male palp of Masikia caliginosa (A-F - Shokalsky Isl.) and M. indistincta (G, H - Dolgiy Isl.). A, B palp, dorsal and retrolateral; C - tibia, dorso-retrolateral; D - tibia, retrolateral; E, G - tibia, dorsal; F, H - tibia, anterior. Scale $=0.05 \mathrm{~mm}$, if not otherwise indicated.

Рис. 6. Пальпа самца Masikia caliginosa (A-F - о. Шокальского) и M. indistincta (G, Н - o. Долгий). А, В — пальпа, сверху и ретролатерально; С — голень, сверху-ретролатерально; D — голень, ретролатерально; Е, G — голень, сверху; F, Н — голень, спереди. Масштаб 0,05 мм, если не обозначено иное.

Masikia caliginosa Millidge, 1984, stat.rev. Figs 1C, D, 2H, 3D-F, 4A-C, 5E-H, 6A-F, 7D-H, 8G-J, 9, Map 1

Masikia caliginosa Millidge,1984: 154, figs 126, 130 (+). Misidentifications (as M. indistincta): Marusik et al., 1992 145 (in part); Eskov, Marusik, 1994: 47 (in part); Tanasevitch, Khrisanova, 2016: 117; Tanasevitch, 2017: 80 (in part); Tanasevitch, Nekhaeva, 2017: 317 (in part).

MATERIAL EXAMINED RUSSIA: Arkhangelsk Area: $20^{7} O^{2}$ 3 우 (ZMMU), Novaya Zemlya Archipelago, Yuzhnyi Isl., nr Bezy-

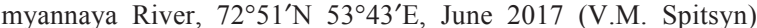
Nenets Autonomous Okrug: $410^{7} 0^{7}$ 88우 (ZMMU), Yugorski Peninsula, env. of Amderma, $69.76^{\circ} \mathrm{N}, 61.67^{\circ} \mathrm{E}$, sea marshes, 4-
8.07.2018 (M.S. Bizin, B.D. Efeikin); $50^{\top} 0^{\top} 2$ 우 (ZMMU), N part of Yamal Peninsula, env. of Sabetta Vil., July 2017, (L.B. Rybalov, A.I. Bastrakov); $470^{7} 0^{7} 7$ 오 (ZMMU), Tazovskii District, Gydan Peninsula, $72.19228^{\circ} \mathrm{N} 77.57839^{\circ} \mathrm{E}$, seashore marshes, $13-$ 18.07.2016 (S.B. Rozenfeld); ca. $2000^{7} \mathrm{o}^{7}+9$ (ZMMU), same district, Shokalsky Isl., $72^{\circ} 58^{\prime} \mathrm{N} 74^{\circ} 27^{\prime} \mathrm{E}$, sea marshes, 8.08.2016 (A.A. Nekhaeva); $10^{7} 1$ ( 9 (ZMMU), Yavai Peninsula, 18.08-1.09. 2013 (M.A. Khrisanova). Krasnoyarsk Province: $220^{7} 0^{7} 590$ (ZMMU), NW Taimyr, $40 \mathrm{~km}$ to $\mathrm{S}$ from Dikson Vil., Efromova River, $73.2006^{\circ} \mathrm{N} 80.8198^{\circ} \mathrm{E}$, moss, July 2003 (D.V. Osipov); $10^{7}$ 2 오 (ZMMU), Taimyr Nature Reserve, Byrranga Mts., 27.07.1992 (A.B. Ryvkin). Yakutia: $40^{7} \sigma^{7}+$ 우, (ZMMU), Ust-Lenskiy Nature Reserve, env. of Tyylaakh, $72.194709^{\circ} \mathrm{N} 128.077528^{\circ} \mathrm{E}, 5-16.07$. 2015 (A.V. Tanasevitch, A.A. Nekhaeva). Chukotka: 1오 (ZMMU), Kresta Bay, env. of Konergino Vil., 3-5.07.1988 (Yu. Marusik); 

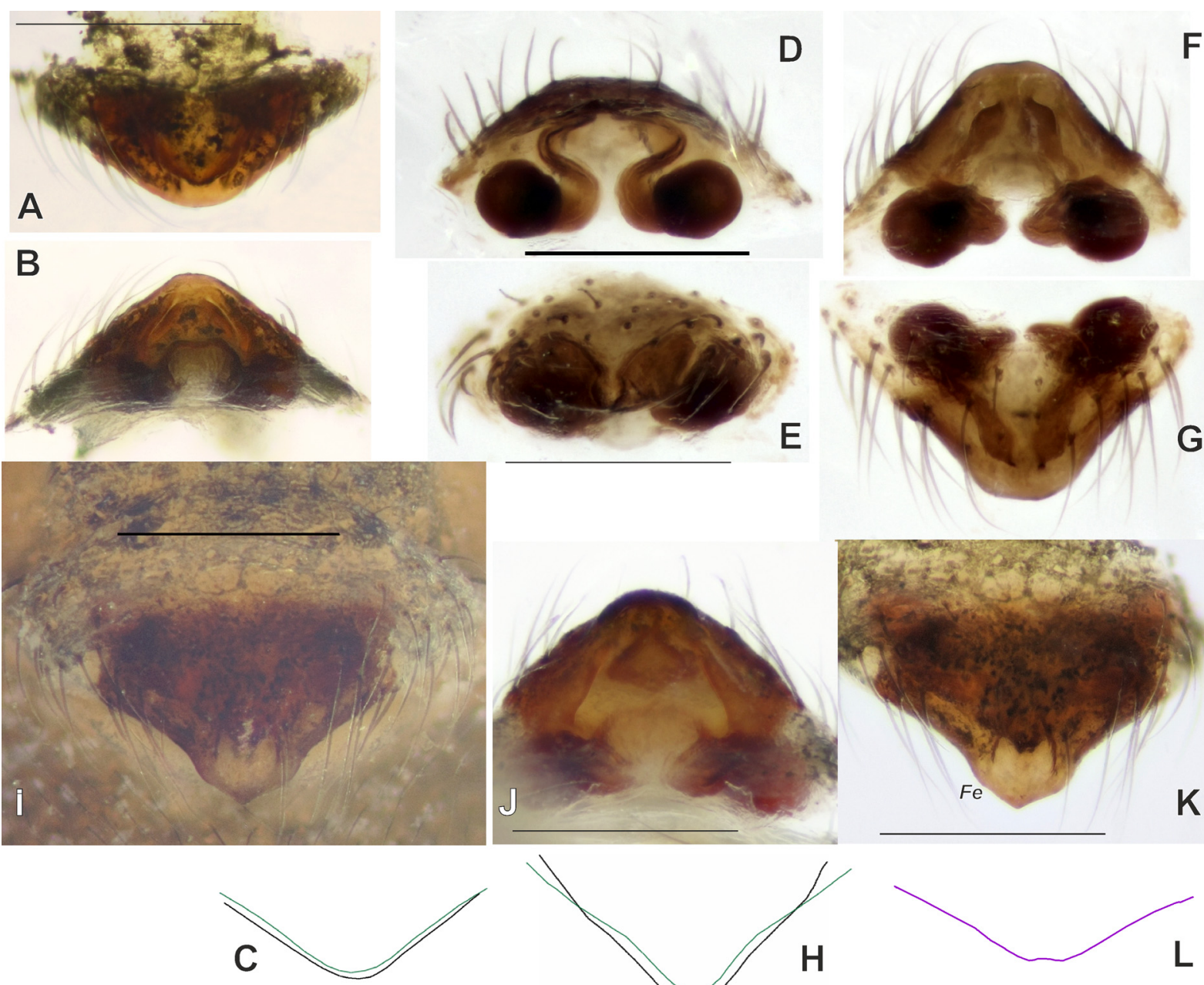

$\mathbf{F}$
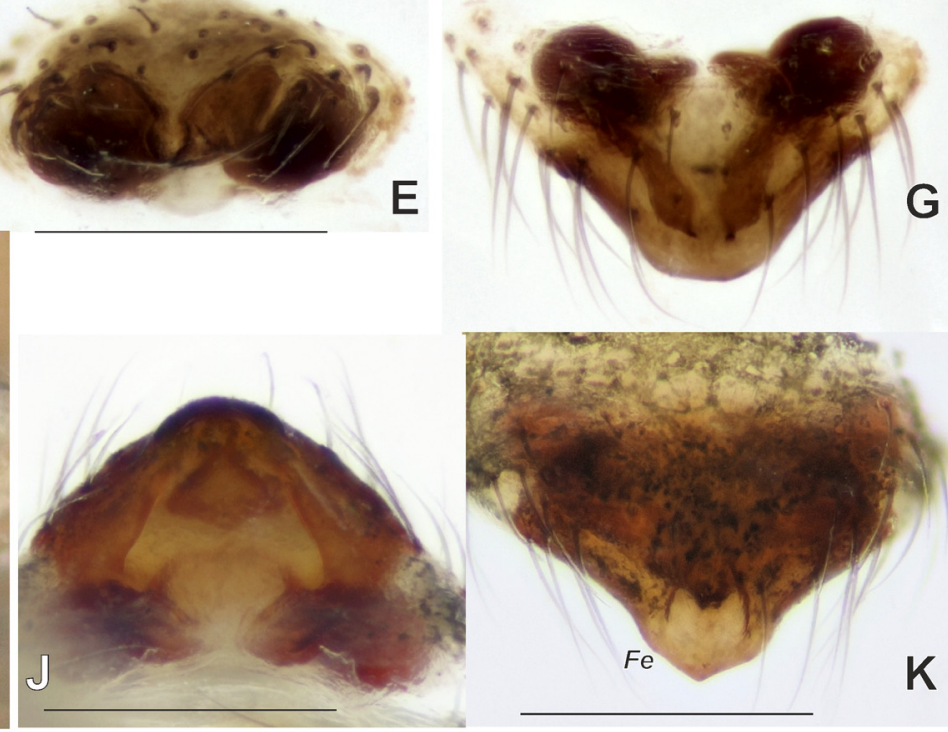

G

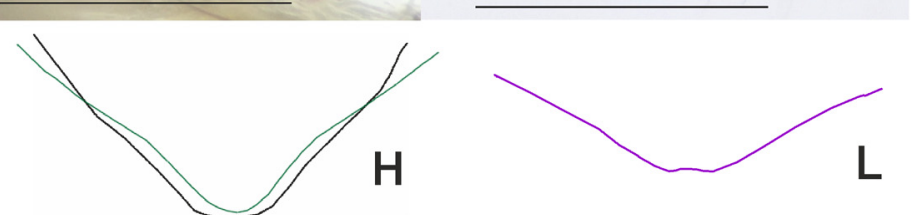

Fig, 7. Epigyne of Masikia indistincta (A-C, Yamal Pnsl.), M. caliginosa (D-H, Shokalski Isl.), M. bizini sp.n. (I-K) and M. relicta (L). A, G, K - dissected, ventral; C, H, L — outline of the ventral view in holotypes (green, violet) and our specimens (black); B, F, J dissected, dorsal; D, E - dissected, anterior and posterior; I - intact, ventral. Scale $=0.2 \mathrm{~mm}$.

Рис. 7. Эпигина Masikia indistincta (A-C, Ямал), M. caliginosa (D-H, о. Шокальского), M. bizini sp.n. (I-K) and M. relicta (L). A, $\mathrm{G}, \mathrm{K}$ - отделенные, снизу; C, H, L — контуры голотипов, снизу (зеленый, фиолетовый) и наших экземпляров (черный); В, F, J отделенные, сверху; D, Е - отделенные, спереди и сзади; I - интактно, снизу. Масштаб 0,2 мм.

1 ( bog, 21.07.2013, leg. A.V. Tanasevitch.

USA: Alaska: $20^{7} 0^{\top} 2$ ㅇํ (DJB), Barrow, 2010 (J. Bowden).

DIAGNOSIS. Males of $M$. caliginosa differ from those of $M$. indistincta by thicker cephalic macrosetae arranged in a bundle (vs. arranged in row), more stridulatory ridges (50 vs. 40). Palps of the two species differ by the shape of the retrolateral and dorsal tibial apophyses (triangle vs. rounded; with deep notch vs. shallow), rounded ventral part of tibia vs. straight; broader embolus (as broad as tailpiece vs. $1 / 2$ of tailpiece width) and shorter stylus. Masikia caliginosa differs from sibling M. bizini sp.n. by smaller size, presence of stylus, shorter terminal part of embolus (cf. Figs $5 \mathrm{~F}$ and $5 \mathrm{~J}$ ), thinner embolus (base of embolus as wide as radix vs. wider than radix).

Females of $M$. caliginosa differ from those of $M$. indistincta by having a more protruding epigynal plate (width/ length ratio 2 vs. 3 ) and having the median plate longer than wide ( $v s$. as long as wide). Epigyne of M. caliginosa differs from that of $M$. bizini sp.n. by its less protruding plate and lack of fenestra (=windows, $\mathrm{Fe}$ ), translucent areas in terminal part of the plate.

DESCRIPTION. Total $\sigma^{7} / 9$ length $1.90-2.3 / 2.03-2.53$. Carapace brown, length/width $0.85-1.0 / 0.7-0.8$ in $\sigma^{7}, 0.88-$

Masikia caliginosa leg lengths.

\begin{tabular}{ccccccc}
\hline $\mathrm{O}^{7} / 9$ & $\mathrm{Fe}$ & $\mathrm{Pa}$ & $\mathrm{Ti}$ & $\mathrm{Me}$ & $\mathrm{Ta}$ & Total \\
\hline I & $0.75 / 0.70$ & $0.23 / 0.25$ & $0.58 / 0.60$ & $0.53 / 0.50$ & $0.43 / 0.43$ & $2.52 / 2.48$ \\
II & $0.73 / 0.65$ & $0.25 / 0.25$ & $0.58 / 0.50$ & $0.53 / 0.50$ & $0.40 / 0.43$ & $2.49 / 2.33$ \\
III & $0.65 / 0.56$ & $0.25 / 0.25$ & $0.50 / 0.48$ & $0.53 / 0.50$ & $0.40 / 0.35$ & $2.33 / 2.14$ \\
IV & $0.88 / 0.83$ & $0.25 / 0.28$ & $0.83 / 0.78$ & $0.78 / 0.75$ & $0.50 / 0.45$ & $3.24 / 3.09$ \\
\hline
\end{tabular}



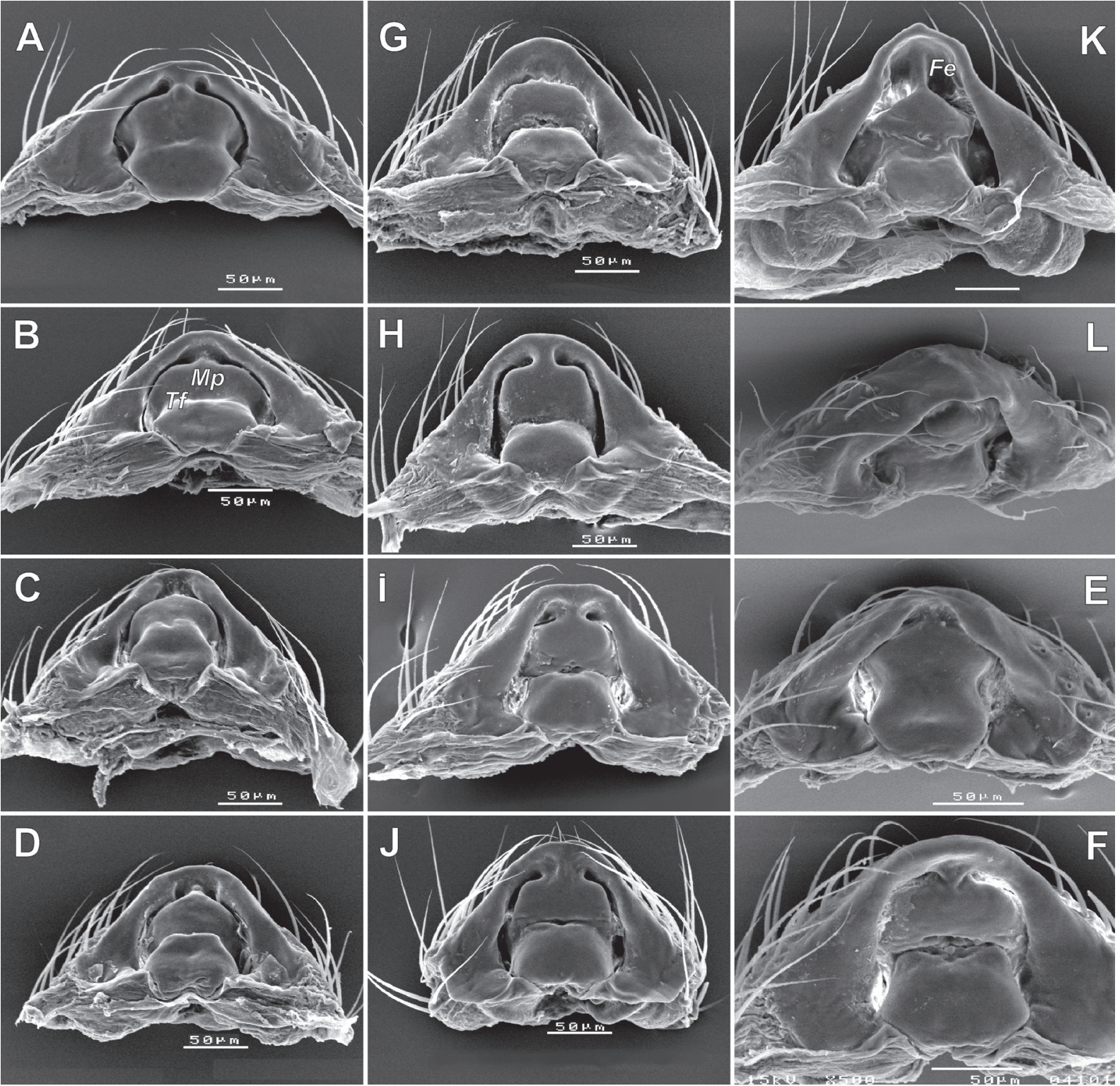

Fig. 8. Epigyne of Masikia indistincta (A-F), M. caliginosa (G-J, from Shokalski Isl.) and M. bizini sp.n. (K, L). A-D, G-J, K dorsal; E, F - posterio-dorsal; L - posterior. A-B - from Dolgiy Isl., C-F - from Pakhancheskaya Guba. Scale $=0.05 \mathrm{~mm}$.

Рис. 8. Эпигина Masikia indistincta (A-F), M. caliginosa (G-J, о. Шокальского) и M. bizini sp.n. (K, L). А-D, G-J, K - сверху; Е, F - сзади-сверху; L - сзади. А-B - c о. Долгий, C-F — из Паханческой Губы. Масштаб 0,05 мм.

$1.13 / 0.6-0.8$ in + . Legs yellowish grey, length of legs as given above; tibial spines 2222 or 2221 (distal spine of TiIV in some specimens absent), TmI 0.5-0.62. Abdomen grey. Male chelicera with about 50 stridulatory ridges (Fig. 3F). Cephalic part in males slightly raised, with 4-5 strong macroseta bases arranged in compact spot and setae in a bundle, length of frontal seta as long as clypeus.

Male palp as in Figs 2H, 4A-C; 5E-H, 6A-F. Tibia with rounded ventral margin; retrolateral apophysis wide triangular; dorsal apophysis with rounded notch, prolateral arm pointed. Embolus broad (as broad as radix), with short stylus; base of embolus imbedded into radical notch.
Epigyne as in Figs 7D-H, 8G-J. Epigynal plate 2 times wider than long; median plate slightly longer than wide.

HABITATS. The species is distributed in the tundra zone and prefers wet habitats. It is one of the dominant species on sea marshes (personal observation) and sedgemoss bogs (D. Osipov, pers. comm.). In several localities, $M$. caliginosa and $M$. indistincta were found to be sympatric, in this case former species numerically prevails over the later (personal observation).

DISTRIBUTION. This species is known from Novaya Zemlya to Cape Barrow, Alaska (Map 1). The southernmost records lie in Chukotka Peninsula $\left(\right.$ ca. $65.55^{\circ} \mathrm{N}$ ), all other records are north of $70^{\circ} \mathrm{N}$. 


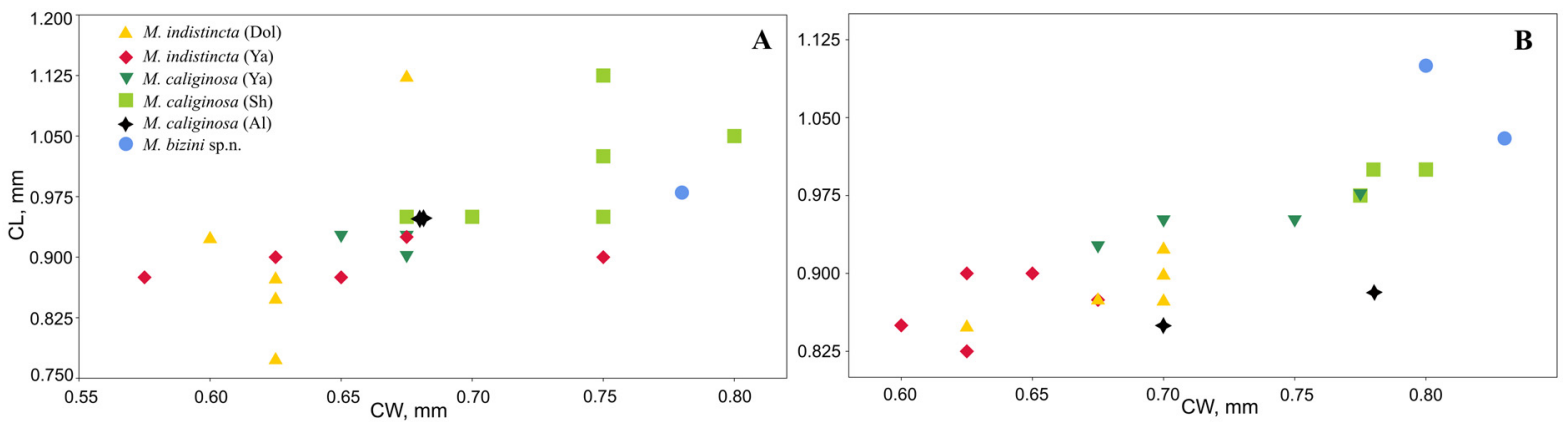

Fig. 9. Carapace length (CL) / width (CW) ratio in three Masikia species. A - females; B - males. Dol - specimens from Dolgiy Isl.; Ya - from Yamal Pnsl.; Al - from Alaska, Barrow, Sh — from Shokalski Isl.

Рис. 9. Отношение длины (CL) к ширине $(\mathrm{CW})$ головогруди у трёх видов Masikia. A — самки; В — самцы. Dol — о. Долгий; Yа — п-в Ямал; Al — Аляска, Барроу, Sh — о. Шокальского.

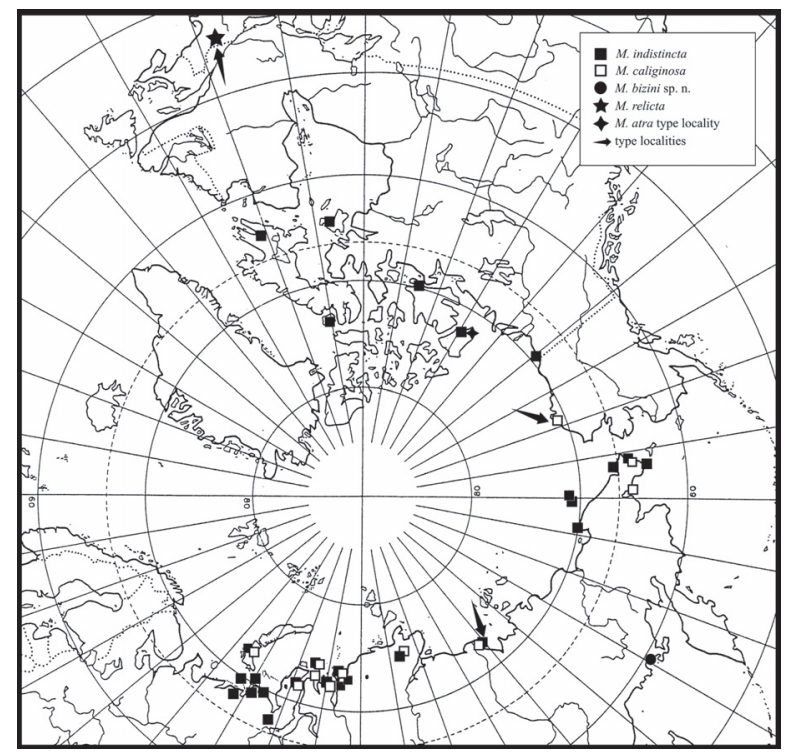

Map 1. Distribution of Masikia species.

Карта 1. Распространение видов рода Masikia.

Masikia bizini sp.n.

Figs 1A, B, 2F, G, 4D-G, 5I-J, 7I-K, 8K, L, 9, Map 1.

TYPES: Holotype $\sigma^{7}$ (ZMMU) and paratypes $10^{7}$ (MMUM) $2 \circ 0$ (ZMMU, MMUM), RUSSIA, NE Siberia, Magadan Area, env. of Tauisk, $59^{\circ} 43^{\prime} 51^{\prime \prime} \mathrm{N} 149^{\circ} 22^{\prime} 47^{\prime \prime} \mathrm{E}$, seashore marshes, pitfall traps, 15-28.07.2017 (M.S. Bizin \& B.D. Efeikin).

DIAGNOSIS. Males of the new species differ from those of $M$. indistincta by stronger cephalic macrosetae arranged in small spot, not a row, larger size (2.08-2.33 vs. 1.851.93), lack of stylus, having elongate tip of embolus and very broad base (wider than tailpiece $v s$. twice as thin). Males of $M$. bizini sp.n. and $M$. caliginosa differ by the shape and width of embolus, and absence vs. presence of stylus. Females of the new species differ from congeners by their much protruding epigynal plate with distinct fenestra, lacking in other species.

DESCRIPTION. Total $\sigma^{7} / q$ length 2.08-2.33/2.48. Carapace brownish, its length/width $1.03-1.1 / 0.80-0.83$ in $\sigma^{7}$, $0.98 / 0.78$ in + . Legs yellowish grey, legs length as shown below; tibial spines 2222 , TmI $0.51-0.55$. Abdomen grey.

Palp as in Figs 2G, 4D-G, 5I, J. Tibia with rounded ventral margin; retrolateral apophysis triangular; dorsal apophysis with shallow notch, both arms with rounded tips. Embolus very broad, wider than radix; its base imbedded into radical notch; stylus absent, tip of embolus long cylindrical.

Epigyne as in Figs 7I-K, 8K, L. Plate strongly protruding, with pointed tip; tip with fenestra (transparent integument); median plate with long and thin stem.

ETYMOLOGY. The species is named after our friend and colleague Mikhail Bizin who collected type material.

HABITAT. The species was collected on sea marshes with a predominance of Puccinellia phryganodes.

DISTRIBUTION. It is known from the type locality only and is the only species occurring in the taiga zone. The other species are distributed either in the tundra zone or found in mountains.

\section{Masikia relicta Chamberlin, 1949} Fig. 7L, Map 1.

Montilaria relicta Chamberlin, 1949: 540, f. 69-70 ().

Masikia relicta: Eskov, Marusik, 1994: 47 (transferred to Masikia).

COMMENTS. This species was described from Mt. Mansfield in Vermont. Its maximal height is only $1339 \mathrm{~m}$, but its top is covered by mountain tundra. The species is known only from the original description and from the holotype female. We have not studied it, because it was out of the scope of our paper. Although the shape of the epigyne of this species is rather similar to those of $M$. indistincta its belonging to Masikia is doubtful. Chamberlin [1949] did

Masikia bizini sp.n. leg lengths.

\begin{tabular}{ccccccc}
\hline $\mathrm{O}^{\top} / q$ & $\mathrm{Fe}$ & $\mathrm{Pa}$ & $\mathrm{Ti}$ & $\mathrm{Me}$ & $\mathrm{Ta}$ & Total \\
\hline I & $0.83 / 0.73$ & $0.28 / 0.23$ & $0.65 / 0.60$ & $0.63 / 0.55$ & $0.48 / 0.35$ & $2.87 / 2.46$ \\
II & $0.75 / 0.70$ & $0.25 / 0.20$ & $0.63 / 0.53$ & $0.58 / 0.45$ & $0.45 / 0.38$ & $2.66 / 2.26$ \\
III & $0.65 / 0.60$ & $0.23 / 0.25$ & $0.55 / 0.48$ & $0.55 / 0.53$ & $0.40 / 0.35$ & $2.38 / 2.21$ \\
IV & $0.93 / 0.85$ & $0.28 / 0.28$ & $0.80 / 0.78$ & $0.80 / 0.70$ & $0.53 / 0.43$ & $3.34 / 3.04$
\end{tabular}


not mention spine formula, the position of trichobothria or the presence or absence of metatarsus IV trichobothrium.

\section{ADDITION}

While the paper was in press new data about Masikia caliginosa distribution were received. Species identification was based on our illustrations.

RUSSIA: Kuril Islands: $2 \sigma^{7} \sigma^{7}, 1 \sigma^{7}$ subad., 2 우 (Burke Museum (UWBM), Seattle, USA), Paramushir Is., $50.052^{\circ} \mathrm{N}, 155$ $359^{\circ} \mathrm{E}, \mathrm{h}=25 \mathrm{~m}$, sifted from Sphagnum moss in young bog close to Bolshaya River, 16.08.1997 (R. Crawford).

\section{Acknowledgments.}

We thank Gergin Blagoev (Guelf, Canada), Joe Bowden (Corner Brook, Canada), Rod Crawford (Seattle, USA), Seppo Koponen (Turku, Finland), Sarah Loboda (Ste-Anne-deBellevue, Canada), Kirill G. Mikhailov (Moscow, Russia), Daniil Osipov (Moscow, Russia) and Andrei V. Tanasevitch (Moscow Russia) for providing us material or information about distribution of Masikia; Ilari E. Sääksjärvi (Turku, Finland) for allowing to use museum facilities, and to anonymous reviewers for valuable comments. The study was supported in part by the Russian Foundation for Basic Research, Project \# 17-04-01603.

\section{References}

Chamberlin R.V. 1949. On some American spiders of the family Erigonidae // Annals of the Entomological Society of America. Vol.41. No.4. P.483-562.

Eskov K.Yu., Marusik Yu.M. 1994. New data on the taxonomy and faunistics of North Asian linyphiid spiders (Aranei Linyphiidae) // Arthropoda Selecta. Vol.2. No.4. P.41-79.
Eskov K.Yu. 1985. [The spiders of tundra-zone in the USSR] // Fauna i ekologiya paukov SSSR. Trudy Zoologicheskogo Instituta AN SSSR. Leningrad. Vol.139. P.121-128 [in Russian].

Kulczyński W. 1908. Araneae et Oribatidae. Expeditionum rossicarum in insulas Novo-Sibiricas annis 1885-1886 et 19001903 susceptarum // Zapiski Imperatorskoi Akademii Nauk St. Petersburg. Ser.8. Vol.18. Fasc.7. P.1-97.

Marusik Yu.M., Koponen S., Makarova O.L. 2016. A survey of spiders (Araneae) collected on the arctic island of Dolgiy $\left(69^{\circ} 12^{\prime} \mathrm{N}\right)$, Barents Sea // Arachnology. Vol.17. No.1. P.10-24.

Marusik Yu.M., Eskov K.Yu., Kim J.P. 1992. A check-list of spiders (Aranei) of Northeast Asia // Korean Arachnology. Vol.8. No.1. P.129-158.

Millidge A.F. 1977. The conformation of the male palpal organs of linyphiid spiders, and its application to the taxonomic and phylogenetic analysis of the family (Araneae: Linyphiidae) // Bulletin of the British Arachnological Society. Vol.4. Pt.1. P.1-60.

Millidge A.F. 1984. The erigonine spiders of North America. Part 7. Miscellaneous genera (Araneae, Linyphiidae) // Journal of Arachnology. Vol.12. No.2. P.121-169.

Paquin P., Buckle D.J., Dupérré N., Dondale C.D. 2010. Checklist of the spiders (Araneae) of Canada and Alaska // Zootaxa. Vol.2461. No.1. P.1-127.

Tanasevitch A.V. 2017. New records of spiders (Aranei) from the Russian Arctic // Arthropoda Selecta. Vol.26. No.1. P.77-82.

Tanasevitch A.V., Khrisanova M.A. 2016. First data on the spiders (Aranei) from the northern Gydan Peninsula, Russia // Arthropoda Selecta. Vol.25. No.1. P.115-118.

Tanasevitch A.V., Nekhaeva A.A. 2016. Spiders (Aranei) of the Kharaulakh Mountains and the Lena River lower reaches, northern Yakutia, Russia // Arthropoda Selecta. Vol.25. No.3. P.307326.

WSC 2019. World Spider Catalog. Version 19.5. Natural History Museum Bern, online at http://wsc.nmbe.ch, accessed on 15.01.2019. doi: $10.24436 / 2$

Responsible editor K.G. Mikhailov 